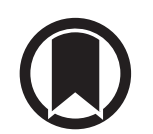

CrossMark

\title{
High-resolution computed tomography features of 17 cases of coronavirus disease 2019 in Sichuan province, China
}

\author{
To the Editor:
}

The city of Wuhan, in Hubei province, China is the focus of global attention due to the coronavirus disease 2019 (COVID-19) outbreak [1]. Sichuan, as a province near Hubei, also has been involved. As of February 12, 2020, 59741 confirmed cases of COVID-19 have been reported in China, of which 451 cases have been identified in Sichuan province. This disease is caused by infection of a new coronavirus named severe acute respiratory syndrome coronavirus 2 (SARS-CoV-2) by the World Health Organization (WHO). According to the latest research, the novel coronavirus is $96 \%$ identical at the whole-genome level to a bat coronavirus, leading to speculation that this new coronavirus may originate from bats $[2,3]$. Current epidemiological data indicate that person-to-person transmission of COVID-19 is occurring [4]. This disease has become a major health crisis in China, and has the potential to become a worldwide epidemic. According to the guidelines stated by the WHO, epidemiological characteristics, clinical manifestations, chest images and laboratory findings represent the major screening tools for identifying COVID-19 infection. Diagnostic confirmation is ultimately dependent on respiratory samples tested by RT-PCR [5]. However, the false-negative rate of RT-PCR is reported to be up to $70 \%$ at the early stage in the clinical course, which may lead to missed diagnosis and thus increased spread of illness. Further, the time required to do the PCR test, given patient flow in the current health crisis and the many other influenza variants that can lead to similar symptoms, can delay treatment and appropriate patient isolation. Therefore, for patients with clinical manifestations and suspect exposure history to the novel coronavirus, clinical imaging findings can play an important role in making preliminary diagnoses and guide patient management decisions.

Currently, limited information exists regarding imaging findings of COVID-19. In this study, we retrospectively analysed high-resolution computed tomography (HRCT) findings in 17 patients suffering from COVID-19 in Sichuan province, China, and discuss how to improve diagnostic accuracy based on these data.

Approval for this study was obtained from the local research ethics committee of West China - Guan'an Hospital of Sichuan University; informed consent was waived.

17 patients with COVID-19 infection confirmed by a positive RT-PCR of sputum samples and admitted to West China - Guang'an Hospital of Sichuan University during the epidemic outbreak in Sichuan province were included.

A multidetector computed tomography scanner (Somatom16; Siemens Healthineers) was employed to obtain chest images. The HRCT scan protocol was as follows: $120 \mathrm{kV}, 150 \mathrm{~mA}$ and $1 \mathrm{~mm}$ slice thickness. The scanning range was from lung apex to diaphragm in the axial plane taken at the end inspiration.

Two thoracic radiologists, with 7 and 11 years of working experience, reviewed the HRCT images independently and resolved discrepancies by consensus. The images were analysed on PACS workstations, using both lung (width, $1500 \mathrm{HU}$; level, $-700 \mathrm{HU}$ ) and mediastinal (width, $350 \mathrm{HU}$; level, $40 \mathrm{HU}$ ) settings.

Our patients included 17 individuals, eight males and nine females, with an age range of 23-74 years (median age, 48.6 years). Time from the onset of symptoms to hospital presentation ranged from 0 to

@ERSpublications

Bilateral ground-glass opacities and a combination of consolidation and ground-glass opacities mainly in the subpleural lung regions is a noteworthy HRCT feature of coronavirus disease 2019, which may help in the early diagnosis of the disease http://bit.ly/2IgXcel

Cite this article as: Zhang S, Li H, Huang S, et al. High-resolution computed tomography features of 17 cases of coronavirus disease 2019 in Sichuan province, China. Eur Respir J 2020; 55: 2000334 [https://doi. org/10.1183/13993003.00334-2020]. 
11 days (median 4.05 days). The main presenting symptoms were fever $(n=12)$, cough $(n=9)$, muscle ache $(n=7)$, sputum production $(n=7)$, fatigue $(n=6)$, chills $(n=5)$, dizziness $(n=4)$, shortness of breath $(n=1)$ and sore throat $(n=1)$. Eight patients had a history of living in Wuhan, five patients had history of contact with proven COVID-19 cases, and four patients had no known contact with any COVID-19 patient. 10 of the 17 patients had no significant medical history. The remaining seven patients had a history of the following comorbidities: hypertension $(n=2)$, chronic gastritis $(n=2)$, rheumatic heart disease $(n=1)$ and COPD $(n=2)$. RT-PCR of all the patients' sputum was positive for the novel coronavirus nucleic acid. All the 17 patients had lymphopenia on presentation. The white blood cell differential count showed that eight patients had increased neutrophils, 15 patients had decreased eosinophils and 16 patients had decreased lymphocytes. All the 17 patients showed elevated blood levels for C-reactive protein and were transferred to infection isolation rooms.

All patients underwent HRCT on the day of admission. The time from symptom onset to performing the initial HRCT ranged from $6 \mathrm{~h}$ to 11 days (median 4.04 days). The initial HRCT scan on the day of admission showed that 12 of 17 patients had ground-glass opacities (GGOs). Five of the 17 patients had a combination of consolidation and GGOs. The distribution of abnormalities was in the subpleural lung regions in 12 patients, bilateral in 14 and unilateral in three of 17 patients. Both upper and lower lobes were involved in 15 patients, only upper lobe was involved in two patients. Air bronchogram was identified in three patients. None of the patients had tree-in-bud pattern, cavitation or pleural effusion. Follow-up HRCT scan were performed in five patients. In three of the five that showed markedly decreased consolidation, fibrotic changes developed (the time intervals between the initial HRCT and follow-up ranged between 6 and 11 days), while the other two patients showed mild progression with increased extent and density of opacities (the time intervals between the initial HRCT and follow-up ranged between 2 and 3 days). Figure 1 shows the HRCT manifestations of four selected cases.

COVID-19 is a new form of atypical pneumonia. It was first reported in the city of Wuhan in December 2019, and has spread rapidly to other areas of China and multiple countries [6]. On January 30, 2020, this

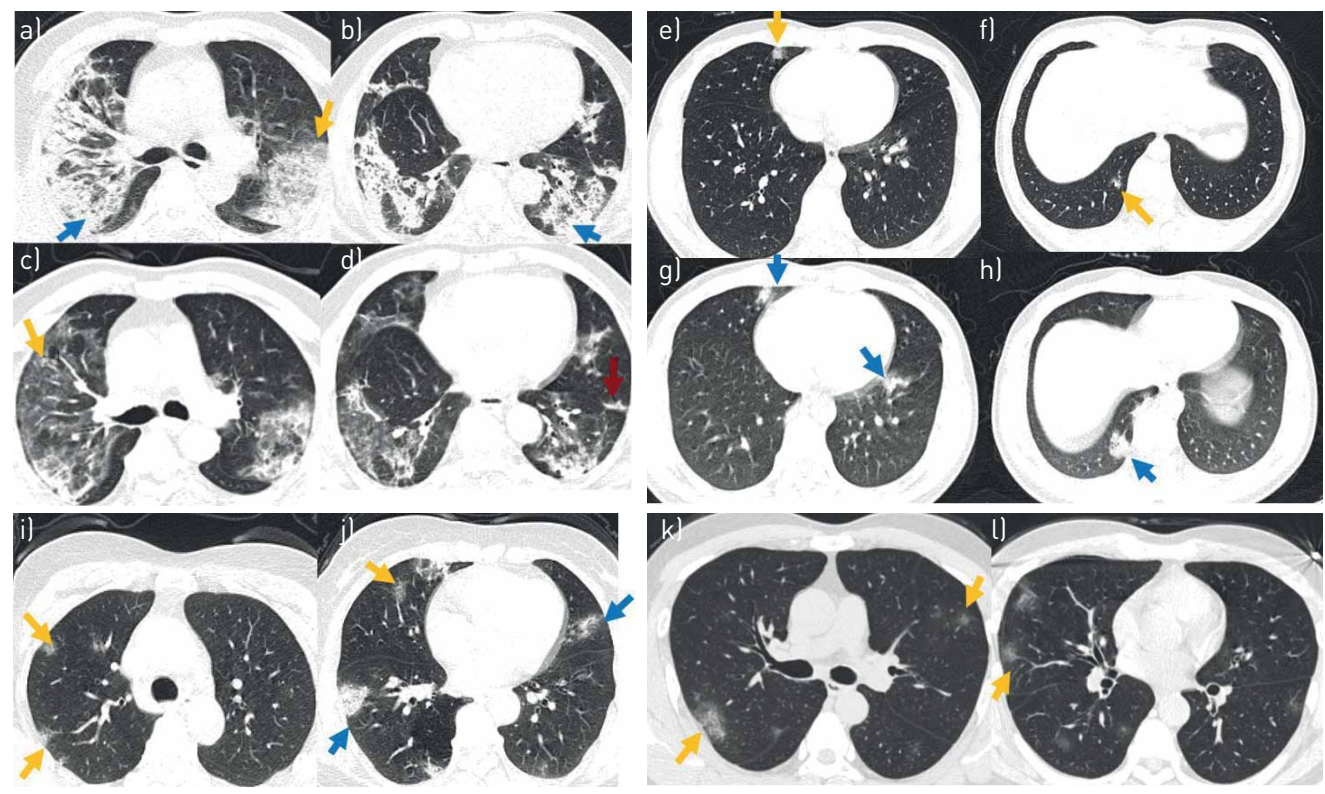

FIGURE 1 High-resolution computed tomography (HRCT) manifestations of selected four cases with coronavirus disease 2019 (COVID-19). Case 1: 57-year-old male patient. HRCT scan was performed on day of admission (8 days after the onset of illness), showing a) a combined pattern of consolidations (blue arrow) and ground-glass opacities (GGOs) (yellow arrow) with air bronchograms in both lobes, and b) areas of consolidation (blue arrow) in both lower lobes. Follow-up HRCT scan performed 10 days after admission, showing $\mathrm{c}$, d) remission of abnormalities, with reduced extent and density of airspace opacification, GGOs (yellow arrows) and fibrosis (red arrow). Case 2: 23-year-old male patient. HRCT scan was performed on day of admission ( $16 \mathrm{~h}$ after the onset of illness), showing e, f) a subpleural distribution of GGOs (yellow arrows) in the middle and lower right lobe. Follow-up HRCT scans performed 3 days after admission show $\mathrm{g}$, hl larger areas of mixed consolidations and GGOs (blue arrows) in the upper right lobe and both lower lobes. Case 3: 50 -year-old female. HRCT scan was performed on the day of admission (4 days after the onset of illness), showing $i, j$ ) a subpleural distribution of GGOs (yellow arrows) and consolidations (blue arrows) in both lobes. Case 4: 43-year-old female. HRCT scan was performed on day of admission (3 days after the onset of illness), showing $k, l$ ) a subpleural distribution of GGOs (yellow arrow) in both lobes. 
new outbreak was declared as a global health emergency by the WHO. Many patients with COVID-19 have developed severe pneumonia, ARDS or multiple organ failure, and died [6]. The main clinical symptoms of our patients were fever, cough, sputum production and muscle ache which were consistent with previous studies, but not diagnostically specific [7].

As GGOs were difficult to detect on chest radiographs, HRCT, as the first-line imaging modality to detect pulmonary changes, is more sensitive in evaluation of the lungs in patients and suspected infections.

The typical findings of initial HRCT in our patients were bilateral GGOs and a combination of bilateral GGOs and consolidation, usually located in the subpleural lung regions. Three patients showed unilateral involvement. Further progression of the disease leads to involvement of other areas within the lung, with more extensive consolidation lesions, suggesting an organising pneumonia pattern of lung injury. During the recovery period, parenchymal scarring has been shown to develop.

Compared with reported cases in severe acute respiratory syndrome (SARS) and Middle East respiratory syndrome (MERS), the HRCT findings of our patients shared relatively specific features including GGOs, consolidation and fibrosis [8-10]. However, the presence and distribution of opacification patterns in our patients was typically multifocal with no upper or lower lobe predominance, which differs from SARS or MERS. SARS commonly has a unifocal presence [11] and MERS predominantly showed basilar lobe distribution [10].

In conclusion, our study suggests that bilateral GGOs and a combination of GGOs and consolidation are the typical features of the COVID-19 patients. The abnormalities predominantly located in the subpleural regions is indicative of an organising pneumonia pattern. Recognising these features and pattern in acutely ill patients with exposure history or other reasons to suspect COVID-19 infection may aid in the rapid identification of likely infection and guide patient management decisions, which can be of great value to speeding treatment and reducing transmission of this communicable disease.

Simin Zhang ${ }^{1,4}$, Huaqiao $\mathrm{Li}^{2,4}$, Songtao Huang ${ }^{2}$, Wei You ${ }^{3}$ and Huaiqiang Sun $\odot^{1}$

${ }^{1}$ Dept of Radiology, West China Hospital of Sichuan University, Chengdu, China. ${ }^{2}$ Dept of Radiology, West China Guang'an Hospital of Sichuan University, Guang'an, China. ${ }^{3}$ Dept of Infectious Diseases, West China - Guang'an Hospital of Sichuan University, Guang'an, China. ${ }^{4}$ These authors contributed equally to this study.

Correspondence: H. Sun, Dept of Radiology, West China Hospital of Sichuan University, No. 37 Guo Xue Xiang, Chengdu 610041, China. E-mail: sunhuaiqiang@scu.edu.cn

Received: 10 Feb 2020 | Accepted after revision: 22 Feb 2020

Support statement: This work was supported by the National Natural Science Foundation of China (grant 91859203) and Young Elite Scientists Sponsorship Program by CAST (YESS20160060). Funding information for this article has been deposited with the Crossref Funder Registry.

Conflict of interest: None declared.

\section{Reference}

1 Zhu N, Zhang D, Wang W, et al. A novel coronavirus from patients with pneumonia in China, 2019. N Engl J Med 2020; 382: 727-733.

2 Zhou P, Yang X-L, Wang X-G, et al. A pneumonia outbreak associated with a new coronavirus of probable bat origin. Nature 2020; 579: 270-273.

3 Wu F, Zhao S, Yu B, et al. A new coronavirus associated with human respiratory disease in China. Nature 2020; 579: 265-269.

4 Chan JF-W, Yuan S, Kok K-H, et al. A familial cluster of pneumonia associated with the 2019 novel coronavirus indicating person-to-person transmission: a study of a family cluster. Lancet 2020; 395: 514-523.

5 Corman VM, Landt O, Kaiser M, et al. Detection of 2019 novel coronavirus (2019-nCoV) by real-time RT-PCR. Eurosurveillance 2020; 25: 2000045.

6 Chen N, Zhou M, Dong X, et al. Epidemiological and clinical characteristics of 99 cases of 2019 novel coronavirus pneumonia in Wuhan, China: a descriptive study. Lancet 2020; 395: 507-513.

7 Huang C, Wang Y, Li X, et al. Clinical features of patients infected with 2019 novel coronavirus in Wuhan, China. Lancet 2020; 395: 497-506.

8 Müller NL, Ooi GC, Khong PL, et al. High-resolution CT findings of severe acute respiratory syndrome at presentation and after admission. AJR Am J Roentgenol 2004; 182: 39-44.

9 Goh JS, Tsou IY, Kaw GJ. Severe acute respiratory syndrome (SARS): imaging findings during the acute and recovery phases of disease. $J$ Thorac Imaging 2003; 18: 195-199.

10 Ajlan AM, Ahyad RA, Jamjoom LG, et al. Middle East respiratory syndrome coronavirus (MERS-CoV) infection: chest CT findings. AJR Am J Roentgenol 2014; 203: 782-787.

11 Wong KT, Antonio GE, Hui DS, et al. Severe acute respiratory syndrome: radiographic appearances and pattern of progression in 138 patients. Radiology 2003; 228: 401-406. 